\title{
COMMENT
}

\section{Host genotype and early life microbiota alterations have additive effects on disease susceptibility}

\author{
Timothy C. Borbet $\mathbb{D}^{1,2}$ and Martin J. Blaser ${ }^{1,2,3}$
}

Why do people develop chronic inflammatory illnesses, such as inflammatory bowel disease and auto-immunity, when they are adults? Is the trigger a recent exposure or may the seeds of the problem have formed much earlier in life? What is the role of genetic susceptibility in such processes?

In this issue of Mucosal Immunology, Goethel et al. (Mucosal Immunol. 1, 2019) developed an experimental system in mice to provide at least partial answers to these important questions, in a model relevant to inflammatory bowel disease (IBD). To examine the role of host genotype, they compared wild type C57BL/6 mice with their congenic NOD2 knockouts, focusing on an important innate immunity gene related to detection of bacterial derived ligands and regulation of inflammatory immune responses (Gut Microbes 4:222-231, 2013). To examine the role of the microbiota, mice were exposed, or not, to amoxicillin, an antibiotic with broad-spectrum activity against the normal constituents of the mammalian intestinal microbiome. Further, to examine developmental phenomena related to host age, mice were exposed either during early life or as adults.

Mucosal Immunology (2019) 12:586-588; https://doi.org/10.1038/s41385-019-0157-1

They found that in the absence of antibiotic treatment, no significant differences were observed in the microbiota composition of wild type and NOD2 knockout mice. This is consistent with prior studies showing that neither NOD1 nor NOD2 deletion impacted microbiota composition. ${ }^{1}$ Other studies of NOD2 knockout embryos transferred into wild type dams, and vice versa, showed NOD2-dependent altered microbiota composition; mice that were deficient in NOD2 signaling or born to NOD2deficient dams had an altered microbiota. ${ }^{2}$ However, horizontal microbiome transfer between cohoused adult mice as a result of coprophagy may diminish the detected microbiome phenotypes associated with genetic knockouts. Such considerations highlight the importance of further definition of NOD2 contributions to intestinal microbiota homeostasis. Goethel et al. ${ }^{3}$ found that with antibiotic treatment, adult NOD2 and wild type mice had comparable perturbations of the microbiota, but after the exposure ceased, NOD2 mice showed more persistent changes in microbiota composition. Early life antibiotic treatment of NOD2 mice increased susceptibility to DSS-colitis and increased the proportion of colonic GATA3 + T regulatory cells relative to other experimental groups, and transfer of the perturbed microbiota to germ-free recipient mice confirmed that it conveyed the enhanced disease signal.

This work brings to light the importance of the experimental design in microbiome studies when genetic knockout models are used, the layered effects of both genotype and insult to the microbiome by antibiotics, and the critical factor of host age and the timing of altered microbiome structure and subsequent disease susceptibility. Each of these points, which are relevant to disease in humans, will be addressed in turn.
First, the importance of breeding and proper controls while conducting a study of the microbiome in genetically modified mice has been well-described; Ubeda et al. ${ }^{4}$ demonstrated how mouse husbandry practices can have a critical influence on the microbiome characteristics in mouse groups, regardless of genotype. Observed differences between wild type and genetic knockout mice may be due to their isolation from one another over multiple generations during breeding, leading to founder effects. The observations of Ubeda et al. ${ }^{4}$ illuminate the importance of heterozygous crosses and studying littermate wild type controls and knockout mice from the F2 generation; this is the optimal approach for reaching sound conclusions and enhancing reproducibility when investigating the microbiome. The impact of the microbiome on host physiological and immunological development, cellular differentiation, and metabolism ${ }^{5-8}$ further emphasize the importance of heterozygous crosses in experiments across all disciplines to avoid additional confounding variables. Using such a study design, the observations of Goethel et al. ${ }^{3}$ are consistent with prior work, and the use of microbiome transfers into a germ-free model to determine the microbiota role in the phenotype provides additional confidence in the study's conclusions.

Second, in considering gene-microbiome interactions, Goethel et al. ${ }^{3}$ highlighted that following the cessation of antibiotic treatment, NOD2 knockout mice showed a more limited return to the pre-antibiotic state (status quo ante) than did the wild type mice. Thus, the compounded selection by the NOD2 genotype and antibiotic exposure reduced the resilience of the microbiota to maintain community structure. In contrast, in the Ubeda et al. study, ${ }^{4}$ mice that had toll-like receptor (TLR) deletions did not have altered microbiota composition, and vancomycin treatment did not

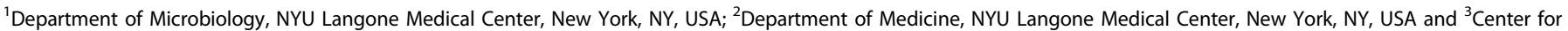
Advanced Biotechnology and Medicine, Rutgers University, New Brunswick, NJ, USA

Correspondence: Martin J. Blaser (martin.blaser@cabm.rutgers.edu)

Received: 10 January 2019 Revised: 22 February 2019 Accepted: 24 February 2019

Published online: 11 March 2019 


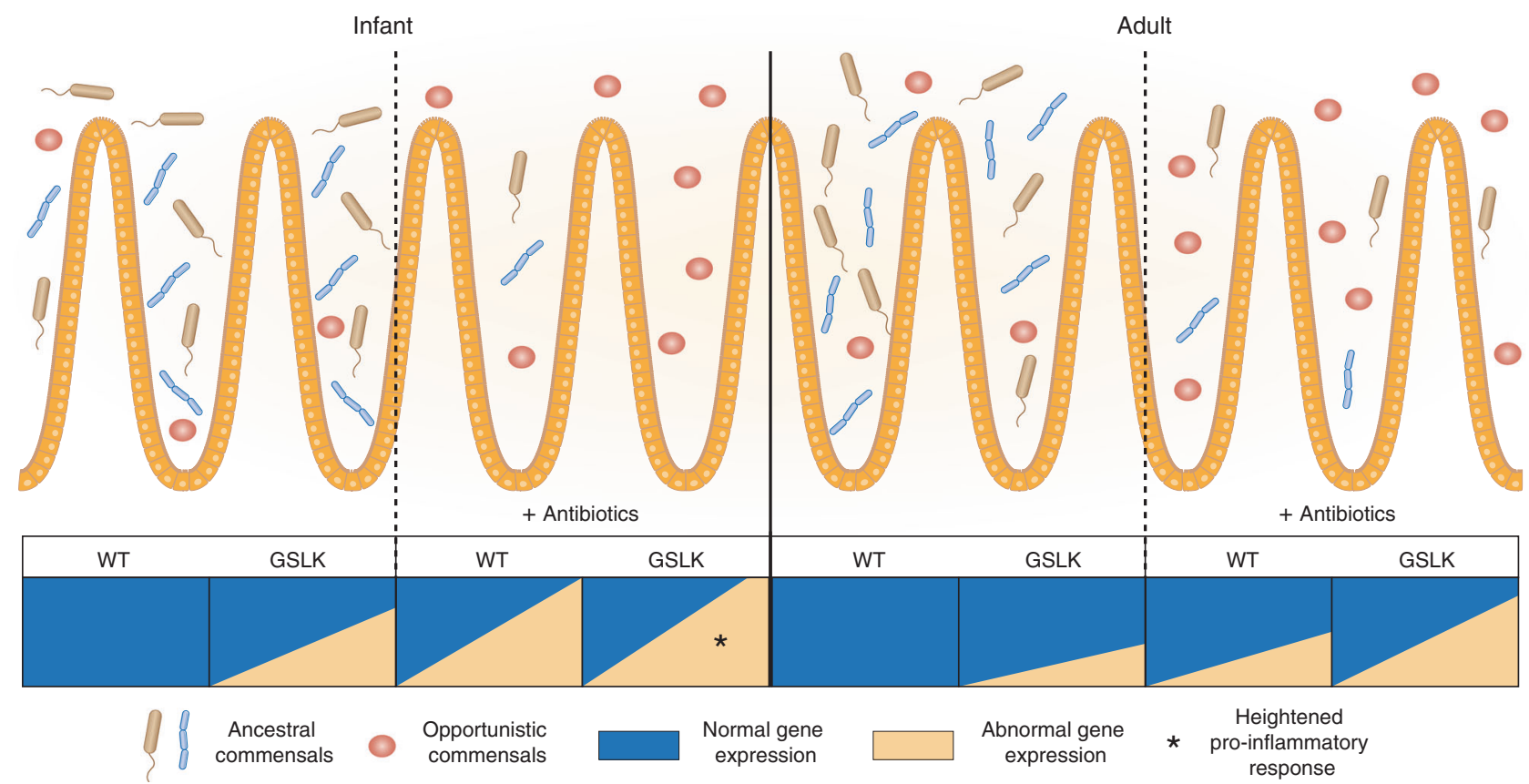

Fig. 1 Model of effects of early life antibiotic-induced microbiome perturbation on host gene expression, immunity, and disease susceptibility. Goethel et al. ${ }^{1}$ contrasted the effects of antibiotic exposure in both infant and adult wild type and genetic susceptibility locus knockout (GSLK) mice. In the NOD2 knockout, immunological and disease susceptibility traits were enhanced in the antibiotic-exposed, infant mice. The adult mice are anticipated to have altered gene expression ${ }^{7}$ without the enhanced susceptibility to disease

affect the resilience of the microbial communities in the knockout mice relative to wild type control littermates. The differences between the studies may reflect the differences between the genes knocked-out (TLRs vs NOD2), the antibiotics used (vancomycin vs. amoxicillin), or the timing of the intervention (7-day treatment of adults vs. the first 21 days of life). Such differences highlight the complexity of the interactions between host and microbiota, and indicate the need for multiple experiments to well-define their characteristics. Because of the complexity of host-microbiome interactions, multiple approaches may be needed to experimentally determine phenotypic variation. ${ }^{9}$ Nevertheless, Goethel et al. ${ }^{3}$ show that host NOD2 receptor signaling plays an important role in selecting microbiome populations, but that provocative perturbations may be needed to elicit the relevant phenotypes.

Third, the clear demonstration that early in life amoxicillin exposure heightened the inflammatory consequences of DSScolitis in the NOD2 mice ${ }^{3}$ highlights the combinatorial effects of specific genes and microbiota perturbations on disease phenotypes. Although the antibiotic provocation, by reshaping the microbiota, can affect disease susceptibility, the effects may not be immediate but can occur after a long window of latency. These findings can guide the evaluation of other host genes that may be important in selecting for intestinal microbial populations that affect resiliency and/or disease susceptibility following microbiota perturbation. Based on these data, future research should focus on other host innate receptors that may affect other diseases linked to microbial perturbations. However, in comparing across studies that use antibiotic exposures to alter the microbiota, both antibiotic class and bioavailability (based on route of delivery, duration, and pharmacodynamics), will need to be considered.

Finally, considerable work is emerging that focuses on the timing of experimental interactions, which indicates that microbiome perturbation during early life is associated with altered immunologic development and disease susceptibility. ${ }^{6-8}$ Such work also indicates that the class of antibiotic used, dose, duration of exposure, and host age during antibiotic exposure can impact how the microbiota is altered as well as the ability for the ecosystem to recover, ${ }^{3,10,11}$ which then affect host responses.
When both pups and dams were exposed to a single 5-day course of a macrolide, the pups showed significantly greater perturbation of ileal gene expression and significant reductions in IL-17 production by CD4 T cells and IgA levels in serum and feces while the dams did not. ${ }^{7}$ These data, in conjunction with the work presented by Goethel et al. ${ }^{3}$ in which only the early life antibiotic treatment with amoxicillin led to enhanced susceptibility to DSScolitis, provide further strong evidence that effects on the microbiota during important developmental windows lead to long-term consequences. ${ }^{11}$ In a world in which human children are highly exposed to antibiotics in the first 2 years of life, ${ }^{12}$ this work suggests sobering implications.

This study and that of others emphasizes the importance of defining the early life interface between the microbiome and the host. That these interactions are truly important is being further substantiated in the context of specific disease states that occur later in life including diabetes, ${ }^{8}$ asthma, ${ }^{5}$ and colitis. ${ }^{3}$ Identifying the microbes that provide strong signals to the host and the host 'sensors' that recognize these microbial cues, will improve the ability to intervene and prevent microbiota-driven disease susceptibility (Fig. 1). Relevant interventions may come in the form of microbial restoration, microbial metabolite supplementation, specified prebiotics, and/or activation of host molecular pathways to facilitate host microbiome resilience. We must better define these critical windows of development in which microbiome alterations have the greatest consequences. Knowing the timing of these windows will have important therapeutic and clinical implications going forward. As we deepen our knowledge of the contributions of the microbiota to host physiology and health, we may discover ways in which we will be able to intervene and lessen disease susceptibility.

\section{AUTHOR CONTRIBUTIONS}

T.C.B. and M.J.B. wrote the manuscript and conceptualized the figure.

\section{ADDITIONAL INFORMATION}

Competing interests: The authors declare no competing interests. 
Host genotype and early life microbiota alterations have additive effects... TC Borbet and M Blaser

588

Publisher's note: Springer Nature remains neutral with regard to jurisdictional claims in published maps and institutional affiliations.

\section{REFERENCES}

1. Robertson, S. J. et al. Nod1 and Nod2 signaling does not alter the composition of intestinal bacterial communities at homeostasis. Gut Microbes 4, 222-231 (2013).

2. Al Nabhani, Z. et al. Nod2 deficiency leads to a specific and transmissible mucosaassociated microbial dysbiosis which is independent of the mucosal barrier defect. J. Crohn's Colitis 10, 1428-1436 (2016).

3. Goethel, A. et al. Nod2 influences microbial resilience and susceptibility to colitis following antibiotic exposure. Mucosal Immunol. 1, (2019). https://doi.org/ 10.1038/s41385-018-0128-y.

4. Ubeda, C. et al. Familial transmission rather than defective innate immunity shapes the distinct intestinal microbiota of TLR-deficient mice. J. Expo. Med. 209, 1445 (2012)
5. Arrieta, M. C. et al. Early infancy microbial and metabolic alterations affect risk of childhood asthma. Sci. Transl. Med. 7, 307ra152-307ra152 (2015).

6. Honda, K. \& Littman, D. R. J. The microbiota in adaptive immune homeostasis and disease. Nature 535, 75 (2016).

7. Ruiz, V. E. et al. A single early-in-life macrolide course has lasting effects on murine microbial network topology and immunity. Nat. Comm. 8, 518 (2017).

8. Zhang, X. S. et al. Antibiotic-induced acceleration of type 1 diabetes alters maturation of innate intestinal immunity. eLife 7, e37816 (2018).

9. Stappenbeck, T. S. \& Virgin, H. W. Accounting for reciprocal host-microbiome interactions in experimental science. Nature 534, 191 (2016).

10. Nobel, Y. R. et al. Metabolic and metagenomic outcomes from early-life pulsed antibiotic treatment. Nat. Comm. 6, 7486 (2015).

11. Cox, L. M. et al. Altering the intestinal microbiota during a critical developmental window has lasting metabolic consequences. Cell 158, 705-721 (2014).

12. Rogawski, E. T. et al. Use of antibiotics in children younger than two years in eight countries: a prospective cohort study. Bull. Who. 95, 49 (2017). 